\section{Stahl- und Gussemailumsätze in 2012 gegenläufig}

Im Geschäftsjahr 2012 hat sich der Absatz von Stahl- und Gussemails gegenläufig entwickelt. Nach Angaben des Deutschen Email Verbandes e.V. (DEV) haben sich im Vergleich zum Vorjahr die Absatz- und Umsatzzahlen bei Stahlemails mengenmäßig um $5,9 \%$, wertmäßig sogar um $14,7 \%$ verringert. Bei Gussemails war der Trend umgekehrt. Mengenmäßig stieg der Absatz um 10,1 \% und wertmäßig um $27 \%$. Insgesamt schlug die Geschäftsentwicklung bei den Stahlemails auf das Gesamtergebnis 2012 durch, da Gussemails nur für Nischen- und Spezialanwendungen eingesetzt werden. Der Marktanteil liegt bei unter $5 \%$. Nach der beim DEV geführten Statistik wurden 20126058 Tonnen Stahlemails abgesetzt mit einem Gesamtumsatz von rund 16,7 Mio. Euro. Von Gussemails wurden 218 Tonnen für rund 1,2 Mio. Euro verkauft. Als Ursache für den deutlich unter Druck geratenen Stahlemailmarkt sieht der Verband die Importe aus Süd- und Südosteuropa.

\section{FreiLacke veranstaltet Systemlack-Forum}

Qaugruppen von Maschinen müsBsen, wenn sie am Endprodukt nebeneinander verbaut werden, hinsichtlich Farbton, Glanz und Struktur möglichst gleich aussehen. Wie das in der Praxis gelingen kann, wird in einer Tagungsveranstaltung aufgezeigt, die der Lackhersteller FreiLacke unter dem Titel "Systemlack Forum" am 5. und 6. Juni 2013 in Donaueschingen veranstaltet. Neben den Fachvorträgen zur Lacktechnik kommen hier auch Anwender zu Wort, die diese Aufgabenstellung in ihren Unternehmen erfolgreich umgesetzt haben. Anlagenbauer berichten beispielsweise darüber, wie die Pulverbeschichtung schwerer, großvolumiger Bauteile oder die Elektrotauchlackierung in diesen Prozess mit eingebunden werden können. Auch für Blechteilefertiger oder Lohnbeschichter ist das Systemlackforum eine gute Möglichkeit, die Aspekte des Zulieferers mit in die Diskussionen einzubringen. Die Veranstaltung richtet sich schwerpunktmäBig an Firmen aus dem Maschinenbau, aus dem Bereich der Bau- und Landmaschinen und an Lohnbeschichter, die zur Fertigstellung ihrer Produkte auf zahlreiche Zulieferfirmen angewiesen sind, bei denen unterschiedlichste Lackierprozesse zur Anwendung kommen.

Weitere Informationen unter Tel. 07707 151-259 oder www.freilacke.de

\section{Sturm-Gruppe gründet Standort in Polen}

ie Sturm Gruppe hat in der polnischen Stadt Rzeszòw einen neuen Standort gegründet. Mariusz Szuminski leitet das Büro, das aktuell unter seiner Führung Projekte im Bereich Fördertechnik für Unternehmen wie Audi, BMW oder MB Türk realisiert. Neun Konstrukteure aus dem Bereich Fördertechnik sollen in Zukunft Aufträge für die Sturm-Gruppe in Polen abwickeln.

\section{Termine}

Reutlingen

16.-17.5.2013

Elektrostatik verstehen und sicher beherrschen

Veranstalter: Schnier Elektrostatik $\mathrm{GmbH}$

www.schnier-elektrostatik.de

Tel. 07121 90973-63

Guangzhou (China)

21.-23.5.2013

SF Expo China 2013

Veranstalter: Wise Exhibition

(Guangdong) Co.,Ltd

www.sf-expo.cn

Tel. +20-37599008

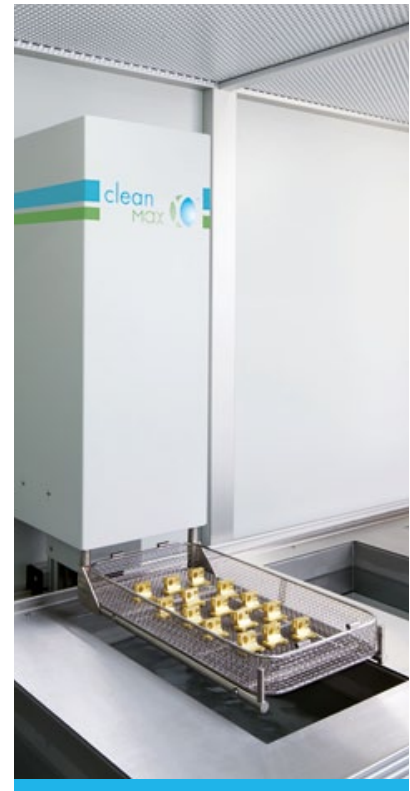

cleanmax

ultrasonic VS1

Ultraschall-

Reinigungsanlage

- sehr kompakte Bauweise

- mind. 4-stufige Reinigung

zzgl. Trocknung

- für sensible und semisensible

Branchen

- für hohe Ansprüche nach

Ihrer DIN

INKLUSIVE:

- Wasseraufbereitung

- vollautom. SPS Steuerung

- Warenbewegung / - transport

- beheizbare Becken

Besuchen Sie uns in

2013 auf folgenden

Messen:

EMO Hannover

16.-21.09.2013

parts2clean

Stuttgart

22.-24.10.2013


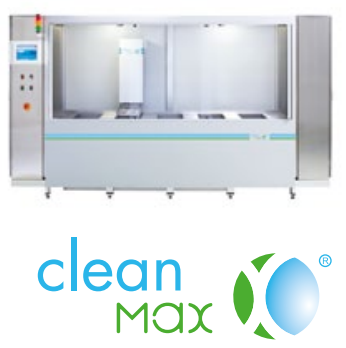

Cleanmax $\mathrm{GmbH}$ Auer Strasse 7, 09366 Stollberg Tel.: +49 37296 92723-600 